\title{
Carbon stock estimation on the reclaimed forest of PT Newmont Minahasa Raya in Ratatotok, Southeast Minahasa
}

H.N. Pollo Forestry Sciences Study Programme, Faculty of Agriculture, Sam Ratulangi University, Indonesia

H.I. Mansur Faculty of Forestry, IPB, Indonesia

C.E.D. Sompie PT Newmont Minahasa Raya, Indonesia

J. Kojansow PT Newmont Minahasa Raya, Indonesia

\begin{abstract}
Describing the quality of forest ecosystems using a carbon sequestration approach is important in understanding global warming. From 1997 to 2010, PT Newmont Minahasa Raya (PTNMR) has reclaimed 215 ha bare land of ex-mining area. The area now has become a fully functioning forest ecosystem. The forest absorbs $\mathrm{CO}_{2}$ from the atmosphere and accumulates it into a living biomass. The aim of this study was to estimate carbon sink in the Ratatotok forest, an ex-mining area. The study observed: 1) species composition at the various stages of plant development; 2) tree height and diameter to estimate the standing biomass; 3) carbon content of the understorey species; 4) carbon content of the ground litters; and 5) soil carbon content. Carbon content measurement employed a loss on ignition method, while total carbon was estimated using existing allometric equations. This study was conducted in the reclaimed areas from six different years, i.e. 1997, 2000, 2001, 2002, 2003 and 2007. The results showed that between 1997 and 2007, the forests captured a total of $247 \mathrm{t}$ carbon per ha, equivalent to a total sequestration of 53,180 $t$ of carbon in the entire reclaimed area (215 ha).
\end{abstract}

\section{$1 \quad$ Introduction}

Reduction of forest cover is an important issue to combat global warming. As forest areas decrease, the capture of $\mathrm{CO}_{2}$ from the atmosphere is also lessened. Local programmes to increase $\mathrm{CO}_{2}$ capture from the atmosphere are essential to combat global warming. PTNMR has committed to reclaim 215 ha of bare land in an ex-mining area in Ratatotok, North Sulawesi, Indonesia, and to restore it to a fully functioning forested ecosystem (Figure 1).

Since rehabilitation, the vegetation has re-established and grown and the area has turned into a more natural ecosystem (Pollo et al., 2011). As trees planted on the area are continuing to grow and capture $\mathrm{CO}_{2}$ from the atmosphere, the forest has retained a certain carbon mass and a certain value of a growing carbon storage that sinks into its carbon pools. Hollinger (2008); Burton and Pregitzer (2008); and Scott and Brown (2008) defined carbon pools as standing trees, leaves, shrubs, litter fall, roots, soil. In order to know how much carbon is sequestered and stocked in the forest, research on carbon stock estimation is important and necessary.

\section{$2 \quad$ Methodology}

Field measurements were carried out on revegetated areas during 1997, 2000, 2001, 2002, 2003, and 2007 (referred to as Rec. 1997 to Rec. 2007). In each revegetated area, three different lines were determined as sampling transects. On each sampling transect, three quadrilateral sampling plots of $20 \times 20 \mathrm{~m}$ were placed for data collecting purpose. All smaller plots were placed systematically at the beginning of the centre line in the plot of $20 \times 20 \mathrm{~m}$. There were 48 plots in total. 


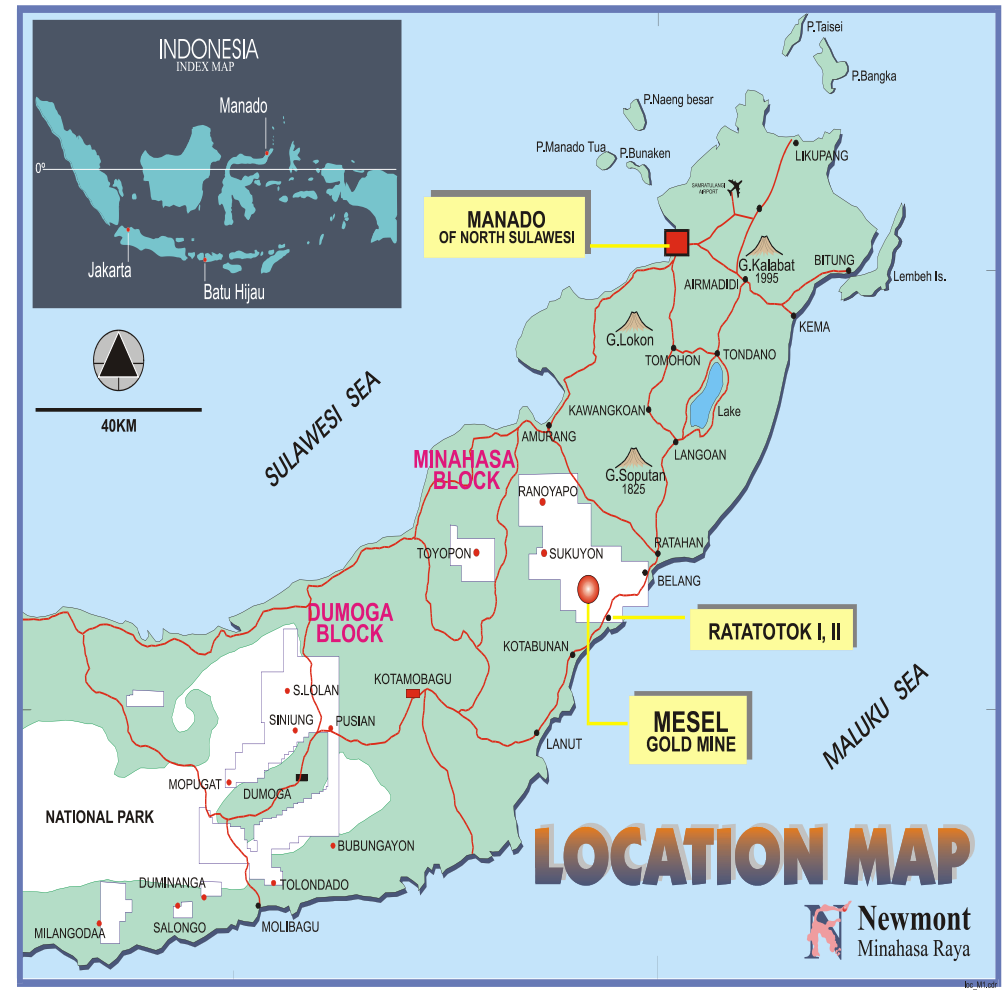

Figure 1 Gold mining location of PTNMR in Ratatotok, Southeast Minahasa (source: PTNMR)

Five parameters are measured in the observation plots:

1. Species composition in various stages of plant development.

2. Tree height and its diameter for biomass calculation on standing trees.

3. Carbon content of understorey (seedlings and ground cover).

4. Carbon content of ground litter.

5. Carbon content of soil.

Tree species and plant life forms were directly identified in the field. The biomass of the standing trees was calculated using allometric equations based on tree diameter which was then multiplied by 0.47 , a default fraction determined by McGroddy et al. (2004) to obtain a carbon fraction value. Carbon content of the understorey vegetation (seedlings and ground cover), ground litter, and on soil were analysed using loss on ignition, combusted in a muffle furnace at $360^{\circ} \mathrm{C}$ for 2 hours, and gravimetric methods (Heiri et al., 2001; Konen et al., 2002). All the work to obtain carbon fraction data was undertaken in the Soil Physics Laboratory of Faculty of Agriculture, Sam Ratulangi University, Indonesia.

\subsection{Species composition at various stages of plant development}

Species composition data were collected from quadrilateral plots which were systematically separated and adjusted directly in each transect. Stages of a plants life cycle consist of seedling, sapling, shrub and pole, and tree. Classification of each stage is as follows:

- Seedlings were classified as herbaceous, semi-woody, and the tree species were identified by diameters $\leq 1 \mathrm{~cm}$ and/or height up to $1 \mathrm{~m}$.

- Sapling diameter was between 1-10 cm; height $>1 \mathrm{~m}$.

- Shrubs and poles diameter was between $10-30 \mathrm{~cm}$.

- Tree diameter was $>30 \mathrm{~cm}$ (following Harlow et al., 1991). 
The plot size for the seedlings was $1 \times 1 \mathrm{~m}$, sapling was $5 \times 5 \mathrm{~m}$, shrub and pole was $10 \times 10 \mathrm{~m}$ and trees were $20 \times 20 \mathrm{~m}$ (Brower and Zar, 1984). All plants found in each plot were recorded and tabulated.

\subsection{Tree height and diameter for biomass calculation on standing tree}

The standing tree for biomass calculation was obtained from sapling, shrub and pole, and tree. The total height of tree was measured using a pole. Tree diameter was measured at breast height with a phi band. Recorded diameter data was then calculated using allometric equations to predict standing tree biomass in $\mathrm{kg} / \mathrm{ha}$ or $\mathrm{t} / \mathrm{ha}$. The biomass value was multiplied by default carbon fraction of 0.47 to obtain carbon value in $\mathrm{kg} / \mathrm{ha}$ or $\mathrm{t} / \mathrm{ha}$. Tropical forest typically contains approximately $47-50 \%$ carbon fraction deposited as terrestrial biomass (Brown, 1997; Eggleston et al., 2006; Chojnacky and Milton, 2008). By adding a time unit, forest productivity levels can be determined.

There are several allometric equations available for biomass calculation as summarised in Table 1.

Table 1 Allometric equations used for biomass calculation

\begin{tabular}{|c|c|c|c|}
\hline No. & Plant Species & Equation & Source \\
\hline \multirow[t]{2}{*}{1} & \multirow{2}{*}{$\begin{array}{l}\text { Paraserienthes } \\
\text { falcataria }\end{array}$} & YAG $=0.3196 D^{1.9834}$ & \multirow[t]{2}{*}{ Krisnawati et al. (2011) } \\
\hline & & $Y R=0.0069 D^{2.5651}$ & \\
\hline \multirow[t]{6}{*}{2} & \multirow{6}{*}{$\begin{array}{l}\text { Swietenia } \\
\text { macrophylla }\end{array}$} & $Y S=0.044 D^{2.61}$ & \multirow{6}{*}{$\begin{array}{l}\text { Adinugroho and Sidiyasa } \\
\text { (2006) }\end{array}$} \\
\hline & & $Y B=0.00059 D^{3.46}$ & \\
\hline & & $\mathrm{YT}=0.0027 \mathrm{D}^{2.42}$ & \\
\hline & & YSt $=0.022 D^{1.96}$ & \\
\hline & & $Y L=0.0138 D^{1.93}$ & \\
\hline & & YTot $=0.048 \mathrm{D}^{2.68}$ & \\
\hline 3 & Gmelina arborea & $Y=0.153 \times D^{2.217}$ & Banaticla et al. (2005) \\
\hline 4 & $\begin{array}{l}\text { Leucaena } \\
\text { leucocephala }\end{array}$ & $Y=0.206 \times D^{2.305}$ & Banaticla et al. (2005) \\
\hline 5 & Tectona grandis & $Y=0.153 \times D^{2.382}$ & Penman et al. (2003) \\
\hline 6 & Tree fern & $A G B=-4266348 /(1-2792284 \exp (-0.313677 \times H))$ & Tiepolo et al. (2002) \\
\hline 7 & $\begin{array}{l}\text { Root biomass } \\
\text { density }\end{array}$ & $Y R=\exp (-1.0587+0.8836 x$ in $A G B)$ & $\begin{array}{l}\text { Cairns et al. (1997) in } \\
\text { Sutaryo (2009) }\end{array}$ \\
\hline 8 & Tropical Rain Forest & $Y=13,2579-4,8945 \times\left(D^{2}\right)+0.6713 \times\left(D^{2}\right)$ & Brown et al. (1989) \\
\hline
\end{tabular}

Notes: $\mathrm{Y}=$ Biomass $(\mathrm{kg}), \mathrm{D}=$ Diameter at Breast Height $(\mathrm{cm}), \mathrm{H}=$ Height $(\mathrm{m}), \mathrm{YAG}=$ Above Ground Biomass, $\mathrm{YS}=$ Stem Biomass, $\mathrm{YB}=\mathrm{Branch}$ Biomass, $Y$ T $=$ Twig Biomass, $Y S t=$ Stump Biomass, $Y \mathrm{~L}=$ Leaf Biomass, $Y$ Tot $=$ Total Above Ground Biomass.

\subsection{Carbon content of understorey vegetation}

Understorey vegetation consists of seedlings and ground cover plants. All understorey living plants, including their roots collected on each plot of $2 \times 2 \mathrm{~m}$, were combusted due to loss of ignition method. The gravimetric method showed that the carbon content of understorey vegetation indicated a loss of combusted mass.

\subsection{Carbon content of ground litter}

Ground litter data was collected on the same plot of $1 \times 1 \mathrm{~m}$ on the seedling's observation plot. Methods employed for carbon content calculation were similar to those that were applied to the understorey category. 


\subsection{Carbon content of soil}

Soil carbon content was obtained by combusting composite soil samples. The soil bulk density value was obtained by oven drying undisturbed soil samples at $105^{\circ} \mathrm{C}$. Composite soil samples were taken from the field in depths of $0-10 \mathrm{~cm}, 10-20 \mathrm{~cm}, 20-30 \mathrm{~cm}$. Undisturbed soil samples were taken in depths of $0-15 \mathrm{~cm}$ and $15-30 \mathrm{~cm}$.

\section{Table 2 Number of seedlings found in all reclaimed areas}

\begin{tabular}{|c|c|c|c|c|c|c|c|c|c|}
\hline No & Species & Rec. 1997 & Rec. 2000 & Rec. 2001 & Rec. 2002 & Rec. 2003 & Rec. 2007 & Total & $\begin{array}{c}\text { Frequency } \\
\text { (\%) }\end{array}$ \\
\hline 1 & Acanthus sp. & & 2 & & & & & 2 & $1.5 \%$ \\
\hline 2 & Ageratum conyzoides & & & 1 & 1 & & & 2 & $1.5 \%$ \\
\hline 3 & Artocarpus sp. & & & & 1 & & & 1 & $0.7 \%$ \\
\hline 5 & Cananga odorata & & 1 & & 2 & 1 & & 4 & $2.9 \%$ \\
\hline 6 & Capsicum frutescens & & & & & 1 & & 1 & $0.7 \%$ \\
\hline 7 & Cardiospermum halicacabum & & & & & & 1 & 1 & $0.7 \%$ \\
\hline 8 & Centrosema pubescens & & & 2 & 5 & & & 7 & $5.1 \%$ \\
\hline 9 & Chromolaena odorata & 1 & 3 & 1 & 1 & & 2 & 8 & $5.9 \%$ \\
\hline 10 & Crotalaria sp. & 1 & 1 & 3 & 5 & 3 & 1 & 14 & $10.3 \%$ \\
\hline 11 & Convolvulaceae & & & & & & 1 & 1 & $0.7 \%$ \\
\hline 12 & Cyathea sp. & & & 1 & 1 & & & 2 & $1.5 \%$ \\
\hline 13 & Ficus septica & & & & & & 1 & 1 & $0.7 \%$ \\
\hline 14 & Ficus sp. (Liana) & & & 1 & & 1 & & 2 & $1.5 \%$ \\
\hline 15 & Ficus sp. 1 & & & 1 & & & & 1 & $0.7 \%$ \\
\hline 16 & Glyrisidia maculata & & & & & 1 & & 1 & $0.7 \%$ \\
\hline 17 & Graminae & & 3 & 4 & 3 & 1 & 2 & 13 & $9.6 \%$ \\
\hline 18 & Hyptis capitata & & 1 & & & & & 1 & $0.7 \%$ \\
\hline 19 & Imperata cylindrica & & & & & & 1 & 1 & $0.7 \%$ \\
\hline 20 & Lauraceae & 1 & & & & & & 1 & $0.7 \%$ \\
\hline 21 & Leucaena glauca & & 1 & 1 & & 3 & & 5 & $3.7 \%$ \\
\hline 22 & Malvaceae & & & & 1 & & & 1 & $0.7 \%$ \\
\hline 23 & Melanolepis multiglandulosa & & 1 & & & & & 1 & $0.7 \%$ \\
\hline 24 & Melothria sp. & & 1 & 1 & & & 1 & 3 & $2.2 \%$ \\
\hline 25 & Mimosa pudica & 1 & & & 5 & & & 6 & $4.4 \%$ \\
\hline 26 & Nephrolepis sp. & 1 & & & & & & 1 & $0.7 \%$ \\
\hline 27 & Paspalum sp. & 1 & 3 & 1 & 6 & & 3 & 14 & $10.3 \%$ \\
\hline 28 & Piper aduncum & & & 1 & & & 2 & 3 & $2.2 \%$ \\
\hline 29 & Piper sp. (Liana) & & 3 & & & & & 3 & $2.2 \%$ \\
\hline 30 & Premna sp. & & & 3 & & 1 & & 4 & $2.9 \%$ \\
\hline 31 & Pteridaceae & 1 & & & & & & 1 & $0.7 \%$ \\
\hline 32 & Pterocarpus indicus & 1 & 1 & & 1 & & & 3 & $2.2 \%$ \\
\hline 33 & Pythecolobium sp. & 2 & & & & & & 2 & $1.5 \%$ \\
\hline 34 & Sida retusa & & & & 1 & & 1 & 2 & $1.5 \%$ \\
\hline 35 & Stachytarpheta jamaicensis & & 1 & & & & & 1 & $0.7 \%$ \\
\hline 36 & Swietenia macrophylla & 3 & 2 & & & & & 5 & $3.7 \%$ \\
\hline 37 & Terminalia catapa & & & & & & 1 & 1 & $0.7 \%$ \\
\hline 38 & Unidentified 1. & & & 1 & & & & 1 & $0.7 \%$ \\
\hline 39 & Unidentified 2. & & & & & 1 & & 1 & $0.7 \%$ \\
\hline 40 & Urena lobata & 1 & 1 & & & & 1 & 3 & $2.2 \%$ \\
\hline 41 & Verbenaceae & & & & & 2 & 8 & 10 & $7.4 \%$ \\
\hline \multirow[t]{3}{*}{42} & Vitis sp. & 1 & & & & & & 1 & $0.7 \%$ \\
\hline & Total & 15 & 25 & 22 & 33 & 15 & 26 & 136 & $100.0 \%$ \\
\hline & Frequency (\%) & $11.0 \%$ & $18.4 \%$ & $16.2 \%$ & $24.3 \%$ & $11.0 \%$ & $19.1 \%$ & $100.0 \%$ & \\
\hline
\end{tabular}




\section{Results and discussion}

\subsection{Species composition at various stages of plant development}

\subsubsection{Seedlings}

Table 2 lists the numbers of seedlings found during field observations. Based on this table, there were several seedling species growing on the same environment to fill the ecological niche. There were at least six plant species found during the observation: creepers, weeds, planted ground covers, shading tree, lianas and the sown plants. Of the 42 plant species found, some grew as weeds, some were planted for a specific purpose, and some species established themselves naturally. Most of them were dispersed into the area by a dispersal agent. One of the most abundant plants found was Crotalaria sp. that was sown specifically as ground cover vegetation.

Table $3 \quad$ Numbers of saplings found in all reclaimed areas

\begin{tabular}{|c|c|c|c|c|c|c|c|c|c|}
\hline No & Species & Rec. 1997 & Rec. 2000 & Rec. 2001 & Rec. 2002 & Rec. 2003 & Rec. 2007 & Total & Frequency \\
\hline 1 & Abutilon sp. & & & 2 & & 1 & 1 & 4 & $1.3 \%$ \\
\hline 2 & Acacia sp. & & 2 & 6 & 16 & & 5 & 29 & $9.1 \%$ \\
\hline 3 & Ailanthus malabarica & & & & & 1 & & 1 & $0.3 \%$ \\
\hline 5 & Cannarium sp. & & & 1 & & & & 1 & $0.3 \%$ \\
\hline 6 & Capsicum frutescens & & & 1 & & & & 1 & $0.3 \%$ \\
\hline 7 & Cassia alata & & & 1 & & & & 1 & $0.3 \%$ \\
\hline 8 & Chromolaena odorata & & & & & & 1 & 1 & $0.3 \%$ \\
\hline 9 & Cinnamomum sp. & 1 & & & & & 1 & 2 & $0.6 \%$ \\
\hline 10 & Cyathea sp. & & & 4 & 3 & 1 & 3 & 11 & $3.4 \%$ \\
\hline 11 & Ficus sp. 1 & & & 1 & & & & 1 & $0.3 \%$ \\
\hline 12 & Ficus sp. 2 & & 1 & & & & & 1 & $0.3 \%$ \\
\hline 13 & Flacourtia sp. & 1 & & & & & & 1 & $0.3 \%$ \\
\hline 14 & Glyrisidia maculata & & 1 & 12 & 5 & 16 & 15 & 49 & $15.4 \%$ \\
\hline 15 & Gmelina arborea & & 22 & & & & & 22 & $6.9 \%$ \\
\hline 16 & Leucaena glauca & & 2 & & & 6 & & 8 & $2.5 \%$ \\
\hline 17 & Macaranga sp. & & & & & & 1 & 1 & $0.3 \%$ \\
\hline 18 & Mangifera $\mathrm{sp}$. & & & 1 & & & & 1 & $0.3 \%$ \\
\hline 19 & Melanolepis multiglandulosa & & 4 & 12 & 2 & & 2 & 20 & $6.3 \%$ \\
\hline 20 & Palaquium sp. & 1 & 2 & 1 & 1 & 1 & 11 & 17 & $5.3 \%$ \\
\hline 21 & Piper aduncum & & & & 2 & & 5 & 7 & $2.2 \%$ \\
\hline 22 & Pometia pinnata & & & & & & 1 & 1 & $0.3 \%$ \\
\hline 23 & Pterocarpus indicus & & & 1 & & & & 1 & $0.3 \%$ \\
\hline 24 & Sapindaceae & & & & 1 & & & 1 & $0.3 \%$ \\
\hline 25 & Stachytarpheta indica & & & & & & 4 & 4 & $1.3 \%$ \\
\hline 26 & Sterculia sp. & 2 & 1 & 2 & & & & 5 & $1.6 \%$ \\
\hline 27 & Swietenia macrophylla & 55 & 16 & 15 & 7 & 6 & 20 & 119 & $37.3 \%$ \\
\hline 28 & Tectona grandis & & & 4 & 2 & & & 6 & $1.9 \%$ \\
\hline \multirow[t]{3}{*}{29} & Urena lobata & & 2 & & 1 & & & 3 & $0.9 \%$ \\
\hline & Total & 60 & 53 & 64 & 40 & 32 & 70 & 319 & $100.0 \%$ \\
\hline & Frequency (\%) & $18.8 \%$ & $16.6 \%$ & $20.1 \%$ & $12.5 \%$ & $10.0 \%$ & $21.9 \%$ & $100.0 \%$ & \\
\hline
\end{tabular}

\subsubsection{Saplings}

As shown on Table 3, from a total of 29 plant species and 319 recorded saplings, all saplings of Swietenia macrophylla and Tectona grandis were the offspring of trees grown in the reclaimed areas. The trees were able to produce fruits, seeds, seedlings and saplings. This implies that the ecological condition of the reclaimed areas were highly suited to the ecological needs of the plants. 


\subsubsection{Shrubs and poles}

Table 4 shows numbers of saplings found during field observation. Based on the data, there were three tree species, namely Glyrisidia maculata, Swietenia macrophylla and Tectona grandis which were growing abundantly within the study site. They were easily identified in the field. These trees are categorised as a fast growing species, with a diameter size between $\geq 10-30 \mathrm{~cm}$. This indicates that the plants have grown well in the reclaimed area

\subsubsection{Trees}

The number of trees found on sampling plots in all reclaimed areas is summarised in Table 5.

As shown on Table 5, there were 105 trees with a diameter $\geq 30-67 \mathrm{~cm}$. The most common plant, as shown on Table 4, was Glyrisidia maculata, a shading tree. This species cannot be seen in the tree form with a diameter $\geq 30 \mathrm{~cm}$. This is most probably caused by lack of light to support its continued development. The most abundant tree was Swietenia macrophylla. Table 6 summarises a combination of all the plants life cycle stages found in the sampling plots by species type.

Table $4 \quad$ Numbers of shrubs and poles found in all reclaimed areas

\begin{tabular}{|c|c|c|c|c|c|c|c|c|c|}
\hline No & Species & Rec. 1997 & Rec. 2000 & Rec. 2001 & Rec. 2002 & Rec. 2003 & Rec. 2007 & Total & Frequency \\
\hline 1 & Paraserianthes falcataria & & 1 & 1 & 4 & & & 6 & $1.8 \%$ \\
\hline 2 & Artocarpus sp. & & & & & & 1 & 1 & $0.3 \%$ \\
\hline 3 & Cyathea sp. & & & 1 & & & 1 & 2 & $0.6 \%$ \\
\hline 4 & Garuga floribunda & & & & 1 & & & 1 & $0.3 \%$ \\
\hline 5 & Glyrisidia maculata & 2 & 5 & 29 & 5 & 14 & 46 & 101 & $30.6 \%$ \\
\hline 6 & Leucaena glauca & & & & & 4 & & 4 & $1.2 \%$ \\
\hline 7 & Macaranga sp. & & & & & & 1 & 1 & $0.3 \%$ \\
\hline 8 & Melanolepis multiglandulosa & & & 1 & & 1 & 4 & 6 & $1.8 \%$ \\
\hline 9 & Melochia sp. & & & & 1 & 1 & 2 & 4 & $1.2 \%$ \\
\hline 10 & Mimusops elengi & 2 & & & & & & 2 & $0.6 \%$ \\
\hline 11 & Palaquium sp. & 1 & & & & & 2 & 3 & $0.9 \%$ \\
\hline 12 & Pterocarpus indicus & & 6 & & & & & 6 & $1.8 \%$ \\
\hline 13 & Spathodea campanulata & & & & 1 & & & 1 & $0.3 \%$ \\
\hline 14 & Pterospermum sp. & & 1 & & & & & 1 & $0.3 \%$ \\
\hline 15 & Swietenia macrophylla & 2 & 18 & 25 & 8 & 33 & 11 & 97 & $29.4 \%$ \\
\hline 16 & Tectona grandis & & 9 & 19 & 44 & 21 & & 93 & $28.2 \%$ \\
\hline \multirow[t]{3}{*}{17} & Trema orientalis & & & & & & 1 & 1 & $0.3 \%$ \\
\hline & Total & 7 & 40 & 76 & 64 & 74 & 69 & 330 & $100.0 \%$ \\
\hline & Frequency & $2.1 \%$ & $12.1 \%$ & $23.0 \%$ & $19.4 \%$ & $22.4 \%$ & $20.9 \%$ & $100.0 \%$ & \\
\hline
\end{tabular}

Table 5 Number of trees found in all reclaimed areas

\begin{tabular}{|c|c|c|c|c|c|c|c|c|c|}
\hline No & Species & Rec. 1997 & Rec. 2000 & Rec. 2001 & Rec. 2002 & Rec. 2003 & Rec. 2007 & Total & Frequency \\
\hline 1 & Paraserianthes falcataria & & 1 & 10 & 9 & 2 & & 22 & $21.0 \%$ \\
\hline 2 & Gmelina arborea & & 8 & & & & & 8 & $7.6 \%$ \\
\hline 3 & Anthocephalus macrophylla & 1 & & & & & & 1 & $1.0 \%$ \\
\hline 4 & Palaquium sp. & 3 & & & & & & 3 & $2.9 \%$ \\
\hline 5 & Pterocarpus indicus & & & & 1 & & & 1 & $1.0 \%$ \\
\hline 6 & Swietenia macrophylla & 25 & 31 & 2 & & & & 58 & $55.2 \%$ \\
\hline 7 & Tectona grandis & & 2 & 3 & 2 & 4 & & 11 & $10.5 \%$ \\
\hline \multirow[t]{3}{*}{8} & Trema orientalis & & & & & 1 & & 1 & $1.0 \%$ \\
\hline & Total & 29 & 42 & 15 & 12 & 7 & 0 & 105 & $100.0 \%$ \\
\hline & Frequency & $27.6 \%$ & $40.0 \%$ & $14.3 \%$ & $11.4 \%$ & $6.7 \%$ & $0.0 \%$ & $100.0 \%$ & \\
\hline
\end{tabular}


Table 6 Numbers of plant life forms found in all reclaimed areas

\begin{tabular}{|c|c|c|c|c|c|c|c|c|c|}
\hline No & Species & Rec. 1997 & Rec. 2000 & Rec. 2001 & Rec. 2002 & Rec. 2003 & Rec. 2007 & Total & Frequency \\
\hline 1 & Abutilon sp. & & & 1 & & 1 & 1 & 3 & $1.6 \%$ \\
\hline 2 & Acacia sp. & & 1 & 1 & 1 & & 1 & 4 & $2.1 \%$ \\
\hline 3 & Acanthus sp. & & 1 & & & & & 1 & $0.5 \%$ \\
\hline 4 & Ageratum conyzoides & & & 1 & 1 & & & 2 & $1.0 \%$ \\
\hline 5 & Ailanthus malabarica & & & & & 1 & & 1 & $0.5 \%$ \\
\hline 6 & Anthocephalus macrophylla & 1 & & & & & & 1 & $0.5 \%$ \\
\hline 7 & Artocarpus sp. & & & & 1 & & 1 & 2 & $1.0 \%$ \\
\hline 8 & Cananga odorata & & 1 & & 1 & 1 & & 3 & $1.6 \%$ \\
\hline 9 & Cannarium sp. & & & 1 & & & & 1 & $0.5 \%$ \\
\hline 10 & Capsicum frutescens & & & 1 & & 1 & & 2 & $1.0 \%$ \\
\hline 11 & Cardiospermum halicacabum & & & & & & 1 & 1 & $0.5 \%$ \\
\hline 12 & Cassia alata & & & 1 & & & & 1 & $0.5 \%$ \\
\hline 13 & Centrosema pubescens & & & 1 & 1 & & & 2 & $1.0 \%$ \\
\hline 14 & Chromolaena odorata & 1 & 1 & 1 & 1 & & 2 & 6 & $3.1 \%$ \\
\hline 15 & Cinnamomum sp. & 1 & & & & & 1 & 2 & $1.0 \%$ \\
\hline 16 & Crotalaria sp. & 1 & 1 & 1 & 1 & 1 & 1 & 6 & $3.1 \%$ \\
\hline 17 & Convolvulaceae & & & & & & 1 & 1 & $0.5 \%$ \\
\hline 18 & Cyathea sp. & & & 3 & 2 & 1 & 2 & 8 & $4.1 \%$ \\
\hline 19 & Ficus septica & & & & & & 1 & 1 & $0.5 \%$ \\
\hline 20 & Ficus sp. (Liana) & & & 1 & & 1 & & 2 & $1.0 \%$ \\
\hline 21 & Ficus sp. 1 & & & 2 & & & & 2 & $1.0 \%$ \\
\hline 22 & Ficus sp. 2 & & 1 & & & & & 1 & $0.5 \%$ \\
\hline 23 & Flacourtia sp. & 1 & & & & & & 1 & $0.5 \%$ \\
\hline 24 & Garuga floribunda & & & & 1 & & & 1 & $0.5 \%$ \\
\hline 25 & Glyrisidia maculata & 1 & 2 & 2 & 2 & 3 & 2 & 12 & $6.2 \%$ \\
\hline 26 & Gmelina arborea & & 2 & & & & & 2 & $1.0 \%$ \\
\hline 27 & Graminae & & 1 & 1 & 1 & 1 & 1 & 5 & $2.6 \%$ \\
\hline 28 & Hyptis capitata & & 1 & & & & & 1 & $0.5 \%$ \\
\hline 29 & Imperata cylindrica & & & & & & 1 & 1 & $0.5 \%$ \\
\hline 30 & Lauraceae & 1 & & & & & & 1 & $0.5 \%$ \\
\hline 31 & Leucaena glauca & & 2 & 1 & & 3 & & 6 & $3.1 \%$ \\
\hline 32 & Macaranga sp. & & & & & & 2 & 2 & $1.0 \%$ \\
\hline 33 & Malvaceae & & & & 1 & & & 1 & $0.5 \%$ \\
\hline 34 & Mangifera sp. & & & 1 & & & & 1 & $0.5 \%$ \\
\hline 35 & Melanolepis multiglandulosa & & 2 & 2 & 1 & 1 & 2 & 8 & $4.1 \%$ \\
\hline 36 & Melochia sp. & & & & 1 & 1 & 1 & 3 & $1.6 \%$ \\
\hline 37 & Melothria sp. & & 1 & 1 & & & 1 & 3 & $1.6 \%$ \\
\hline 38 & Mimosa pudica & 1 & & & 1 & & & 2 & $1.0 \%$ \\
\hline 39 & Mimusops elengi & 1 & & & & & & 1 & $0.5 \%$ \\
\hline 40 & Nephrolepis sp. & 1 & & & & & & 1 & $0.5 \%$ \\
\hline 41 & Palaquium sp. & 3 & 1 & 1 & 1 & 1 & 2 & 9 & $4.7 \%$ \\
\hline 42 & Paraserianthes falcataria & & 2 & 2 & 2 & 1 & & 7 & $3.6 \%$ \\
\hline 43 & Paspalum sp. & 1 & 1 & 1 & 1 & & 1 & 5 & $2.6 \%$ \\
\hline 44 & Piper aduncum & & & 1 & 1 & & 2 & 4 & $2.1 \%$ \\
\hline 45 & Piper sp. (Liana) & & 1 & & & & & 1 & $0.5 \%$ \\
\hline 46 & Pometia pinnata & & & & & & 1 & 1 & $0.5 \%$ \\
\hline 47 & Premna sp. & & & 1 & & 1 & & 2 & $1.0 \%$ \\
\hline 48 & Pteridaceae & 1 & & & & & & 1 & $0.5 \%$ \\
\hline 49 & Pterocarpus indicus & 1 & 2 & 1 & 2 & & & 6 & $3.1 \%$ \\
\hline 50 & Pterospermum sp. & & 1 & & & & & 1 & $0.5 \%$ \\
\hline 51 & Pythecolobium sp. & 2 & & & & & & 2 & $1.0 \%$ \\
\hline 52 & Sapindaceae & & & & 1 & & & 1 & $0.5 \%$ \\
\hline
\end{tabular}


Table 6 Numbers of plant life forms found in all reclaimed areas continued...

\begin{tabular}{|c|c|c|c|c|c|c|c|c|c|}
\hline No & Species & Rec. 1997 & Rec. 2000 & Rec. 2001 & Rec. 2002 & Rec. 2003 & Rec. 2007 & Total & Frequency \\
\hline 53 & Sida retusa & & & & 1 & & 1 & 2 & $1.0 \%$ \\
\hline 54 & Spathodea campanulata & & & & 1 & & & 1 & $0.5 \%$ \\
\hline 55 & Stachytarpheta indica & & & & & & 1 & 1 & $0.5 \%$ \\
\hline 56 & Stachytarpheta jamaicensis & & 1 & & & & & 1 & $0.5 \%$ \\
\hline 57 & Sterculia sp. & 1 & 1 & 1 & & & & 3 & $1.6 \%$ \\
\hline 58 & Swietenia macrophylla & 4 & 4 & 3 & 2 & 2 & 2 & 17 & $8.8 \%$ \\
\hline 59 & Tectona grandis & & 2 & 3 & 3 & 2 & & 10 & $5.2 \%$ \\
\hline 60 & Terminalia catapa & & & & & & 1 & 1 & $0.5 \%$ \\
\hline 61 & Trema orientalis & & & & & 1 & 1 & 2 & $1.0 \%$ \\
\hline 62 & Unidentified 1. & & & 1 & & & & 1 & $0.5 \%$ \\
\hline 63 & Unidentified 2. & & & & & 1 & & 1 & $0.5 \%$ \\
\hline 64 & Urena lobata & 1 & 2 & & 1 & & 1 & 5 & $2.6 \%$ \\
\hline 65 & Verbenaceae & & & & & 1 & 1 & 2 & $1.0 \%$ \\
\hline \multirow[t]{3}{*}{66} & Vitis sp. & 1 & & & & & & 1 & $0.5 \%$ \\
\hline & Total & 25 & 35 & 38 & 33 & 26 & 36 & 193 & $100.0 \%$ \\
\hline & Frequency & $13.0 \%$ & $18.1 \%$ & $19.7 \%$ & $17.1 \%$ & $13.5 \%$ & $18.7 \%$ & $100.0 \%$ & \\
\hline
\end{tabular}

As shown on Table 6, from the 66 plant species found in all sampling plots, there were four common species namely, Crotalaria sp., Glyrisidia maculata, Palaquium sp. and Swietenia macrophylla. Crotalaria sp. is a species used as a ground cover to protect bare soil from erosion. This pioneer species was planted while waiting for other tree species to form a forested area. Glyrisidia maculata was planted to serve as a shading tree to reduce sunlight intensity. This species was also planted to create suitable microclimate surrounding each main tree to allow the trees to cover the reclaimed areas. Palaquium sp. and Swietenia macrophylla were planted as the main tree vegetation in the reclaimed area.

\subsection{Carbon content of standing trees}

Table 7 shows that there were 753 trees with diameter $\geq 10-67 \mathrm{~cm}$ on a combined total of $19,200 \mathrm{~m}^{2}$ of sampling plots. If the value was converted to numbers of trees per ha, then the total number of trees per ha is 392 , or 84,280 trees in an area of 215 ha. This number of trees in a commercial plantation would be considered of reasonable economic value. The biggest average tree diameter was found in the plots located in the reclaimed year of 2000. Since it was located on a hilly area, it was different from the branching free trees found on the 2002 reclaimed area which is situated on flat ground and where, on average, the tree growth reached $3.63 \mathrm{~m}$. In general, the location of the tree considerably influenced its growth quality.

As shown on Table 7, there were six tree species which reached a diameter corresponding to the largest growth stage. These are considered fast growing species. Anthocephalus macrophyllus and Trema orientalis naturally invaded the reclaimed areas with an annual growth increment of 4.45 and $3.7 \mathrm{~cm} /$ year, respectively. The fastest growth increment was found in Paraserianthes falcataria with $5.18 \mathrm{~cm} /$ year during 2003.

Table 8 summarises the biomass and carbon contents in $\mathrm{kg} / \mathrm{ha}$ on roots and standing trees.

As shown on Table 8, an average sum of root and standing carbon content was $240,513 \mathrm{~kg} / \mathrm{ha}$. In general, carbon content of the reclaimed forest of year 2000 was the highest compared to the others. It was even higher than the trees planted in 1997. This fits with the above explanation.

Based on research done in Indonesia by Dharmawan and Siregar (2009) in Masripatin et al. (2010), and Samsoedin et al. (2009) in Masripatin et al. (2010), above ground carbon stock in a natural lowland tropical rainforest with a diameter at breast height ranging from $7-70 \mathrm{~cm}$ was $230.10-264.70 \mathrm{t} / \mathrm{ha}$. This research found that the standing stock biomass was $435,326 \mathrm{~kg} / \mathrm{ha}$ or $204,603 \mathrm{~kg} / \mathrm{ha}$ of carbon or $205 \mathrm{t} / \mathrm{ha}$ of carbon which indicated that the reclaimed forest functions similar to that of a natural forest. 
Table 7 Averages of height, diameter and branch-free bole length of trees found on sampling plots

\begin{tabular}{|c|c|c|c|c|c|c|c|}
\hline No. & Area & $\begin{array}{c}\Sigma \\
\text { Trees }\end{array}$ & $\begin{array}{l}\text { Diameter } \\
(\mathbf{c m})\end{array}$ & $\begin{array}{l}\text { Height } \\
\text { (m) }\end{array}$ & $\begin{array}{l}\text { Branching } \\
\text { Free }(m)\end{array}$ & $\begin{array}{l}\text { Largest Diameter } \\
(\mathrm{cm})\end{array}$ & Tallest (m) \\
\hline 1 & Rec. 1997 & 96 & 14.02 & 8.37 & 2.37 & $\begin{array}{r}67 \text { (Anthocephalus } \\
\text { macrophyllus) }\end{array}$ & $\begin{array}{l}30.25 \text { (Palaquium } \\
\text { sp.) }\end{array}$ \\
\hline 2 & Rec. 2000 & 134 & 19.09 & 11.45 & 3.33 & $\begin{array}{l}45.5 \text { (Swietenia } \\
\text { macrophylla) }\end{array}$ & $\begin{array}{c}26.5 \text { (Gmelina } \\
\text { arborea) }\end{array}$ \\
\hline 3 & Rec. 2001 & 155 & 13.62 & 9.70 & 2.85 & $\begin{array}{l}43.8 \text { (Paraserianthes } \\
\text { falcataria) }\end{array}$ & $\begin{array}{c}26 \text { (Paraserianthes } \\
\text { falcataria) }\end{array}$ \\
\hline 4 & Rec. 2002 & 116 & 14.94 & 10.93 & 3.63 & $\begin{array}{c}36 \text { (Paraserianthes } \\
\text { falcataria) }\end{array}$ & $\begin{array}{c}24.5 \text { (Paraserianthes } \\
\text { falcataria) }\end{array}$ \\
\hline 5 & Rec. 2003 & 113 & 13.79 & 10.19 & 3.28 & $\begin{array}{l}46.6 \text { (Paraserianthes } \\
\text { falcataria) }\end{array}$ & 22 (Trema orientalis) \\
\hline \multirow[t]{3}{*}{6} & Rec. 2007 & 139 & 8.63 & 7.75 & 2.34 & $\begin{array}{l}18.5 \text { (Trema } \\
\text { orientalis) }\end{array}$ & $\begin{array}{l}15.5 \text { (Trema } \\
\text { orientalis) }\end{array}$ \\
\hline & Total & 753 & 84.09 & 58.39 & 17.81 & & \\
\hline & Average & 126 & 14.01 & 9.73 & 2.97 & & \\
\hline
\end{tabular}

Table 8 Root and standing tree biomass and carbon content of standing trees

\begin{tabular}{|c|c|c|c|c|c|c|c|c|c|}
\hline No. & Area & $\begin{array}{l}\text { Width } \\
\text { (ha) }\end{array}$ & $\underset{\text { Trees }}{\boldsymbol{\Sigma}}$ & $\begin{array}{c}\text { Root } \\
\text { Biomass } \\
\text { (kg/ha) }\end{array}$ & $\begin{array}{c}\text { Standing } \\
\text { Biomass } \\
\text { (kg/ha) }\end{array}$ & $\begin{array}{c}\Sigma \\
\text { Biomass } \\
\text { (kg/ha) }\end{array}$ & $\begin{array}{c}\text { Root to } \\
\text { Standing } \\
\text { Biomass } \\
(\%)\end{array}$ & $\begin{array}{l}\text { Carbon } \\
(\mathrm{kg} / \mathrm{ha})\end{array}$ & $\begin{array}{l}\text { Total } \\
\text { Carbon } \\
(t)\end{array}$ \\
\hline 1 & Rec. 1997 & 16.4 & 96 & 93,457 & 585,173 & 678,630 & 15.97 & 318,956 & 5,321 \\
\hline 2 & Rec. 2000 & 16.5 & 134 & 137,601 & 818,324 & 955,926 & 16.81 & 449,285 & 7,413 \\
\hline 3 & Rec. 2001 & 54.71 & 155 & 78,339 & 424,918 & 503,256 & 18.44 & 236,531 & 12,941 \\
\hline 4 & Rec. 2002 & 51.58 & 116 & 66,197 & 367,783 & 433,980 & 18.00 & 203,971 & 10,521 \\
\hline 5 & Rec. 2003 & 7.0 & 113 & 57,237 & 300,722 & 357,959 & 19.03 & 168,241 & 1,178 \\
\hline \multirow[t]{3}{*}{6} & Rec. 2007 & 68.81 & 139 & 25,594 & 115,035 & 140,629 & 22.25 & 66,096 & 4,548 \\
\hline & Total & 215 & 753 & 458,424 & $2,611,955$ & $3,070,380$ & 110.50 & $1,443,078$ & 41,831 \\
\hline & Average & 35.83 & 126 & 76,404 & 435,326 & 511,730 & 18.42 & 240,513 & 6,972 \\
\hline
\end{tabular}

However to the contrary, the ratio between root and standing biomass was inverted, the younger the vegetation, the higher the value. This can be explained by the fact that soil formation is slower than that of root penetration into a deeper soil solum.

\subsection{Carbon content of understorey}

Table 8 shows an average sum of understorey carbon content of $240,513 \mathrm{~kg} / \mathrm{ha}$. As shown in the data, carbon content of understorey in 2007 was the highest. As this area was reclaimed within five years, the tree canopy had just started to grow. Therefore, there were still gaps among vegetation which allowed the sunlight to reach the forest floor. This created a niche for other plants to grow. Not surprisingly, the carbon content of this area was the lowest. The soil condition of this area was also poor as it had a high clay 
content. This affected the drainage and aeration. During the rainy season, the water inundates the area. Therefore, only plants that are tolerant to high moisture survive, especially during a long rainy season. Typically, weed plant species have a wide range of tolerance to this particular ecological condition.

Table 9 Carbon content of the understorey

\begin{tabular}{ccccccc}
\hline No. & Area & $\begin{array}{c}\text { Width } \\
\text { (ha) }\end{array}$ & $\begin{array}{c}\text { Dry Weight } \\
\text { (kg) }\end{array}$ & $\begin{array}{c}\text { Dry Weight } \\
\text { Average (kg) }\end{array}$ & $\begin{array}{c}\text { Dry Weight } \\
\text { (kg/ha) }\end{array}$ & $\begin{array}{c}\text { Total } \\
\text { Carbon (t) }\end{array}$ \\
\hline 1 & Rec. 1997 & 16.4 & 0.83 & 0.275 & 2,750 & 45 \\
2 & Rec. 2000 & 16.5 & 1.81 & 0.2007 & 2,007 & 33 \\
3 & Rec. 2001 & 54.71 & 4.46 & 0.4955 & 4,955 & 271 \\
4 & Rec. 2002 & 51.58 & 5.74 & 0.6381 & 6,381 & 329 \\
5 & Rec. 2003 & 7 & 0.98 & 0.1087 & 1,087 & 8 \\
6 & Rec. 2007 & 68.81 & 8.69 & 0.9657 & 9,657 & 665 \\
\hline & Total & $\mathbf{2 1 5}$ & $\mathbf{2 2 . 5}$ & $\mathbf{2 . 6 8}$ & $\mathbf{2 6 , 8 3 7}$ & $\mathbf{1 , 5 3 1}$ \\
\hline & Average & $\mathbf{3 5 . 8 3}$ & $\mathbf{0 . 4 7}$ & $\mathbf{0 . 4 5}$ & $\mathbf{4 , 4 7 3}$ & $\mathbf{2 2 5}$ \\
\hline
\end{tabular}

\subsection{Carbon content of ground litter}

Table 10 shows carbon content of the ground litter. Based on the data, an average sum of carbon content was $8,845 \mathrm{~kg} / \mathrm{ha}$. There were two areas producing high carbon content, the reclaimed areas of 1997 and 2002. These two areas had high litter decomposition as the vegetation had formed a dense canopy. The litters were produced primarily by a deciduous tree, Tectona grandis.

Table 10 Carbon content of the ground litter

\begin{tabular}{rrccccc}
\hline No. & Area & $\begin{array}{c}\text { Width } \\
\text { (ha) }\end{array}$ & $\begin{array}{c}\text { Dry Weight } \\
\text { (kg) }\end{array}$ & $\begin{array}{c}\text { Dry Weight } \\
\text { Average (kg) }\end{array}$ & $\begin{array}{c}\text { Dry Weight } \\
\text { (kg/ha) }\end{array}$ & $\begin{array}{c}\text { Total } \\
\text { Carbon (t) }\end{array}$ \\
\hline 1 & Rec. 1997 & 16.4 & 4.03 & 1.3445 & 13,445 & 221 \\
2 & Rec. 2000 & 16.5 & 8.03 & 0.8917 & 8,917 & 147 \\
3 & Rec. 2001 & 54.71 & 6.25 & 0.6941 & 6,941 & 380 \\
4 & Rec. 2002 & 51.58 & 9.69 & 1.0769 & 10,769 & 556 \\
5 & Rec. 2003 & 7 & 6.61 & 0.7343 & 7,343 & 51 \\
6 & Rec. 2007 & 68.81 & 5.09 & 0.5653 & 5,653 & 389 \\
\hline & Total & $\mathbf{2 1 5}$ & $\mathbf{3 9 . 6 9}$ & $\mathbf{5 . 3 1}$ & $\mathbf{5 3 , 0 6 8}$ & $\mathbf{1 , 7 4 3}$ \\
\hline & Average & $\mathbf{3 5 . 8 3}$ & $\mathbf{6 . 6 2}$ & $\mathbf{0 . 8 8}$ & $\mathbf{8 , 8 4 5}$ & $\mathbf{2 9 1}$ \\
\hline
\end{tabular}

\subsection{Carbon content of soil}

Based on the Table 11, the years with the highest dry weight were 1997 and 2007, when the average sum of soil carbon content was 46.38 and $41.52 \mathrm{~kg} / \mathrm{ha}$ respectively.

The carbon distribution in tropical forest ecosystems is different to more temperate regions. The majority of carbon in the tropics is accumulated at the standing biomass, with only a small fraction found in the soil as a result of the more rapid decomposition in a tropical climate (Tasirin, 1994). In 1997 the high carbon content can be explained as a result of high soil retention in addition to the age of plants. High soil carbon 
content in 2007 was probably due to a high rate of decomposition and availability of understorey to supply organic carbon to the soil.

Table 11 Carbon content of soil

\begin{tabular}{cccccccc}
\hline No. & Area & $\begin{array}{c}\text { Width } \\
\text { (ha) }\end{array}$ & $\begin{array}{c}\text { Dry Weight } \\
\text { (gr) }\end{array}$ & $\begin{array}{c}\text { Dry Weight } \\
\text { Average (gr) }\end{array}$ & $\begin{array}{c}\text { Dry Weight } \\
\text { (gr/ha) }\end{array}$ & $\begin{array}{c}\text { Dry Weight } \\
\text { (kg/ha) }\end{array}$ & $\begin{array}{c}\text { Total } \\
\text { Carbon (kg) }\end{array}$ \\
\hline 1 & Rec. 1997 & 16.4 & 13.9127 & 4.6376 & 46,376 & 46.38 & 761 \\
2 & Rec. 2000 & 16.5 & 8.0007 & 2.6669 & 26,669 & 26.67 & 440 \\
3 & Rec. 2001 & 54.71 & 34.2972 & 3.8108 & 38,108 & 38.11 & 2,085 \\
4 & Rec. 2002 & 51.58 & 32.1785 & 3.5754 & 35,754 & 35.75 & 1,844 \\
5 & Rec. 2003 & 7 & 34.4813 & 3.8313 & 38,313 & 38.31 & 268 \\
6 & Rec. 2007 & 68.81 & 37.3661 & 4.1518 & 41,518 & 41.52 & 2,857 \\
\hline & Total & $\mathbf{2 1 5}$ & $\mathbf{1 6 0 . 2 3 6 5}$ & $\mathbf{2 2 . 6 7 3 7}$ & $\mathbf{2 2 6 , 7 3 7}$ & $\mathbf{2 2 6 . 7 4}$ & $\mathbf{8 , 2 5 5}$ \\
\hline & Average & $\mathbf{3 5 . 8 3}$ & $\mathbf{2 6 . 7 0 6 1}$ & $\mathbf{3 . 7 7 8 9}$ & $\mathbf{3 7 , 7 8 9}$ & $\mathbf{3 7 . 7 9}$ & $\mathbf{1 , 3 7 6}$ \\
\hline
\end{tabular}

Table 12 shows a total carbon content in the reclaimed forest. Values in the table were summarised from Tables 8-11.

Table 12 Carbon content in the reclaimed forest

\begin{tabular}{|c|c|c|c|c|c|c|c|c|}
\hline \multirow[b]{2}{*}{ No. } & \multirow[b]{2}{*}{ Area } & \multirow[b]{2}{*}{$\begin{array}{l}\text { Width } \\
\text { (ha) }\end{array}$} & \multicolumn{4}{|c|}{ Carbon Content (t/215 ha) } & \multirow[b]{2}{*}{$\begin{array}{l}\text { Carbon } \\
\text { (t/215 ha) }\end{array}$} & \multirow[b]{2}{*}{$\begin{array}{l}\text { Carbon } \\
\text { (t/ha) }\end{array}$} \\
\hline & & & $\begin{array}{l}\text { Standing } \\
\text { Trees }\end{array}$ & Understorey & $\begin{array}{l}\text { Ground } \\
\text { Litter }\end{array}$ & Soil & & \\
\hline 1 & Rec. 1997 & 16.4 & 5,321 & 45 & 221 & 761 & 6,257 & 29 \\
\hline 2 & Rec. 2000 & 16.5 & 7,413 & 33 & 147 & 440 & 8,033 & 37 \\
\hline 3 & Rec. 2001 & 54.71 & 12,941 & 271 & 380 & 2,085 & 15,676 & 73 \\
\hline 4 & Rec. 2002 & 51.58 & 10,521 & 329 & 556 & 1,844 & 13,250 & 62 \\
\hline 5 & Rec. 2003 & 7 & 1,178 & 8 & 51 & 268 & 1,505 & 7 \\
\hline \multirow[t]{3}{*}{6} & Rec. 2007 & 68.81 & 4,548 & 665 & 389 & 2,857 & 8,458 & 39 \\
\hline & Total & 215 & 41,831 & 1,531 & 1,743 & 8,255 & 53,180 & 247 \\
\hline & Average & 35.83 & 6,972 & 225 & 291 & 1,376 & 8,863 & 41 \\
\hline
\end{tabular}

Based on Table 12 data, it is shown that the carbon content of the reclaimed forest was 247 tonne/ha or in total, the forest stored 53,180 t of carbon per 215 ha. This indicated that the forest has sequestrated $\mathrm{CO}_{2}$ quickly from the atmosphere. The growing forest ecosystem continues to store carbon into the carbon pools. These findings imply that the forest ecosystem has a potential economic value for carbon storage.

\section{$4 \quad$ Conclusion}

Within 5-15 years after planting, trees on the reclaimed forests captured a total of $247 \mathrm{t}$ carbon/ha, or a total sink of 53,180 t of organic carbon in the 215 ha, and the forest performed as a carbon storage. 


\section{References}

Adinugroho, W.C. and Sidiyasa, K. (2006) Model Pendugaan Biomassa Pohon Mahoni (Swietenia macrophyla King) di Atas Permukaan Tanah, Jurnal penelitian Hutan dan Konservasi Alam, Vol. III, No. 1, pp. 103-117.

Banaticla, M.R.N., Sales, R.F. and Lasco, R.D. (2005) Biomass Equations for Tropical Tree Plantation Species Using Secondary Data from the Philippines, in ACIAR Smallholder Forestry Project - Redevelopment of a Timber Industry Following Extensive Land Clearing: Proceedings from the End-of-Project Workshop, S.R. Harrison, J.L. Herbohn, Suh, Jungho, Mangaoang, Eduardo and J. Vanclay (eds), Ormoc City, Philippines, 19-21 August, 2004, pp. 113-128.

Brower, J.E. and Zar, J.A. (1984) Field and Laboratory Methods for General Ecology, 2nd edition, Wm. C. Brown Pub, Dubuque, lowa, $226 \mathrm{p}$.

Brown, S. (1997) Estimating Biomass and Biomass Change of Tropical Forests: a Primer, (FAO Forestry Paper - 134), FAO, Rome, viewed 12 May 2012, http://www.fao.org/docrep/W4095E/W4095E00.htm.

Brown, S., Gillespie, A.J.R. and Lugo, A.E. (1989) Biomass Estimation Methods for Tropical Forests with Applications to Forest Inventory Data, Forest Science, The Society American Foresters, Vol. 35, No. 4, pp. 881-902.

Burton, A.J. and Pregitzer, K.S. (2008) Measuring Forest Floor, Mineral Soil, and Root Carbon Stocks, Part IV, in Field Measurements for Forest Carbon Monitoring: A Landscape-Scale Approach, C.M. Hoover (ed), Springer Science + Business Media B.V. (2008), Chapter 10, 240 p.

Chojnacky, D.C. and Milton, M. (2008) Measuring Carbon in Shrubs, Part II, in Field Measurements for Forest Carbon Monitoring: A Landscape-Scale Approach, C.M. Hoover (ed), Springer Science + Business Media B.V. (2008), Chapter 5, 240 p.

Eggleston, S., Buendia, L., Miwa, K. and Tanabe, K. (eds) (2006) IPPC Guidlines for National Greenhouse Gas Inventories, Vol. 4, Agriculture, Forestry and Other Land Use, The Institute for Global Environmental Strategies (IGES) for The Intergovernmental Panel on Climate Change (IPCC), Hayama, Kanagawa, Japan, $83 \mathrm{p}$.

Harlow, W.M., Harrar, E.S., Hardin, J.W. and White, F.M. (1991) Textbook of Dendrology, 7th Edition, McGraw-Hill Inc., Singapore, $501 \mathrm{p}$.

Heiri, O., Lotter, A.F. and Lemcke, G. (2001) Loss on Ignition as a Method for Estimating Organic and Carbonate Content in Sediments: Reproducibility and Comparability of Results, Journal of Paleolimnology, Kluwer Academic Publishers, The Netherlands, Vol. 25, pp. 101-110.

Hollinger, D.Y. (2008) Defining a Landscape-Scale Monitoring Tier for the North American Carbon Program, Part I, in Field Measurements for Forest Carbon Monitoring: A Landscape-Scale Approach, C.M. Hoover (ed), Springer Science + Business Media B.V. (2008), Chapter 1, 240 p.

Konen, M.E., Jacobs, P.M., Burras, C.L., Talaga, B.J. and Mason, J.A. (2002) Equations for Predicting Soil Organic Carbon Using LossOn-Ignition for North Central U.S. Soils, Soil Science Society of America Journal, Vol. 66, pp. 1878-1881.

Krisnawati, H., Varis, E., Kallio, M. and Kanninen, M. (2011) Paraserienthes falcataria (L.) Nielsen: Ekologi, Silvikultur dan Produktivitas, Center for International Forestry Research, Bogor, Indonesia, $14 \mathrm{p}$.

Masripatin, N., Ginoga, K., Pari, G., Dharmawan, W.S., Siregar, C.A., Ari Wibowo, Puspasari, D., Utomo, A.S., Sakuntaladewi, N., Lugina, M., Indartik, Wulandari, W., Darmawan, S., Heryansah, I., Heriyanto, N.M., Siringoringo, H.H., Damayanti, R., Anggraeni, D., Krisnawati, H., Maryani, R., Apriyanto, D. and Subekti, B. (2010) Carbon Stocks of Various Types of Forest and Vegetation in Indonesia, Center for Climate Change and Policy Research and Development (Pusat Penelitian dan Pengembangan Perubahan Iklim dan Kebijakan), Bogor, Indonesia, 43 p.

McGroddy, M.E., Daufresne, T. and Hedin, L.O. (2004) Scaling of C:N:P stoichiometry in forests worldwide: Implications of terrestrial Redfield-type ratios, Ecology, Vol. 85(9), pp. 2390-2401.

Penman, J., Gytarsky, M., Hiraishi, T., Krug, T., Kruger, D., Riitta Pipatti, Buendia, L., Miwa, K., Ngara, T., Tanabe, K. and Wagner, F. (eds) (2003) Good Practice Guidance for Land Use, Land-Use Change and Forestry, IPCC National Greenhouse Gas Inventories Programme, The Institute for Global Environmental Strategies (IGES) for The Intergovernmental Panel on Climate Change (IPCC), Hayama, Kanagawa, Japan, 625 p.

Pollo, H.N., Sompie, D., Kojansow, J. and Mansur, H.I. (2011) Reclamation and Its Succession in the Used Mine Site of PT Newmont Minahasa Raya in Ratatotok, Southeast Minahasa - A Case Study, in Proceedings Sixth International Conference on Mine Closure (Mine Closure 2011), A.B. Fourie, M. Tibbett and A. Beersing (eds), 19-21 September 2011, Lake Louise, Canada, Australian Centre for Geomechanics, Perth, Vol. 1, pp. 281-290.

Scott, N.A. and Brown, S. (2008) Measuring the Decomposition of Down Dead-Wood, Part III, in Field Measurements for Forest Carbon Monitoring: A Landscape-Scale Approach, C.M. Hoover (ed), Springer Science + Business Media B.V. (2008), Chapter 9, $240 \mathrm{p}$.

Sutaryo, D. (2009) Penghitungan Biomassa: Sebuah Pengantar untuk Studi Karbon dan Perdagangan Karbon, Wetlands International Indonesia Programme, Bogor, $39 \mathrm{p.}$

Tasirin, J.S. (1994) The Role of Raspberry in Nutrient Cycling in a Successional Northern Hardwood Forest Ecosystem, Thesis, University of New Brunswick, Canada.

Tiepolo, G., Calmon, M. and Feretti, A.R. (2002) Measuring and Monitoring Carbon Stocks at the Guaraqueçaba Climate Action Project, Paraná, Brazil, International Symposium on Forest Carbon Sequestration and Monitoring, Taiwan Forestry Research Institute, Extension Series, No. 153, pp. 98-115. 